\title{
Abundância e flutuação populacional das espécies de Chrysomya (Diptera, Calliphoridae) em Pelotas, Rio Grande do Sul, Brasil
}

\author{
Élvia E. S. Vianna ${ }^{1}$, Paulo R. P. Costa ${ }^{2}$, Ana Lúcia Fernandes ${ }^{3,4} \&$ Paulo B. Ribeiro ${ }^{3}$ \\ 1. Laboratório de Entomologia, Museu de História Natural, Universidade Católica de Pelotas, Rua Félix da Cunha, 412, 96010-000 \\ Pelotas, RS, Brasil. (eevianna@phoenix.ucpel.tche.br) \\ 2. In memoriam. \\ 3. Depto de Microbiologia e Parasitologia, Instituto de Biologia, Universidade Federal de Pelotas, 96010-900 Pelotas, RS, Brasil. \\ 4. Bolsista PIBIC (CNPq).
}

\begin{abstract}
Abundance and populational fluctuation of Chrysomya (Diptera, Calliphoridae) species, in Pelotas, Rio Grande do Sul, Brazil. To estimate the populational fluctuation of Chrysomya Robineau-Desvoidy, 1830 species and the relation of populational abundance around, six wind oriented trap (WOT) were placed in three distinct ecological areas (urban, rural and wild) in Pelotas, Rio Grande do Sul, Brazil, from February/1993 to January/ 1995. The flies were weekly collected. Captured species were Chrysomya albiceps Wiedmann, 1819, C. megacephala Fabricius, 1794 and C. putoria Wiedmann, 1830 with respective abundance of $64.5 \%, 19.7 \%$ and $0.9 \%$, representing a total of $85.0 \%$ of 409,920 specimens of Calliphoridae. The three species demonstrated similarity in the populational fluctuation, except in the abundance. The populational peak ocurred in autum when the temperature decreases. In the months of July to November no fly was collected, recomposing the population in December, when the temperature surpassed $20^{\circ} \mathrm{C}$.
\end{abstract}

KEYWORDS. Population fluctuation, Chrysomya, Calliphoridae.

\section{INTRODUÇÃO}

As espécies de Chrysomya Robineau-Desvoidy, 1830 exercem grande importância na saúde pública, não só pela possibilidade de causarem miíases, mas principalmente como vetores mecânicos de patógenos, decorrentes do comportamento de oviposição (sobre carcaças, vísceras de suínos e de aves, lixões urbanos, fossas sépticas e fezes) e alto grau de sinantropia (PATTON, 1922; ZUMPT, 1965; GUIMARÃES et al.1978; BAUMGARTNER \& GreEnBERG, 1984; Vianna et al., 1997). Os califorídeos, apesar de causadores de miíases facultativas (OliveIRA, 1982) e veiculadores de patógenos (GREENBERG, 1973), auxiliam na investigação médico-criminal, permitindo a estimativa do intervalo post-mortem e a detecção de drogas e toxinas ante-mortem (CATTS \& GOFF, 1992), além de contribuírem na natureza como polinizadores e decompositores de cadáveres. Relevantes estudos têm apresentado subsídios à compreensão das relações interespecíficas de califorídeos durante o processo de sucessão em cadáveres, auxiliando no desenvolvimento e implementação de práticas de controle destes dípteros (Von Zuben et al., 1993; Moura et al.,1997).

Os fatores bióticos e abióticos são responsáveis pela flutuação e composição das populações de muscóideos sinantrópicos (NuORTEVA, 1963; DAJOZ, 1983). Fatores bióticos podem obscurecer o efeito direto de fatores climáticos na incidência desses muscóideos em áreas metropolitanas (STEWART \& RoESLER, 1942). Essa incidência está intimamente relacionada à sazonalidade, como demonstram SCHOOF et al. (1954), FerReIRA (1978, 1983) e LiNHARES (1981).

A flutuação de uma população é determinada pelo afastamento assimétrico do nível de equilíbrio da mesma e as populações de insetos flutuam em função do tempo devido à ação de fatores ecológicos (SILVEIRA-NeTo et al., 1976). Assim, diferentes espécies de muscóideos são regidas por fatores ambientais e suas populações alteradas em função das diferentes épocas do ano (VIANNA et al., 1997).

$\mathrm{Na}$ impossibilidade de estimar-se a densidade absoluta de insetos, recorre-se a outras alternativas como a abundância e flutuação populacional, que permitem avaliar as populações no tempo e no espaço.

O objetivo é estimar a abundância e a flutuação populacional das espécies de Chrysomya, relacionando com a temperatura e precipitação pluviométrica médias mensais em Pelotas, Rio Grande do Sul, Brasil.

\section{MATERIAL E MÉTODOS}

Os califorídeos foram capturados em seis armadilhas orientadas pelo vento (WOT, Wind Oriented Trap), construídas de acordo com modelo de Broce et al. (1977) modificado por Oliveira (1982) e instaladas em três áreas ecológicas (urbana, rural e silvestre), em Pelotas (31'52'00"S, 52 21'24'W, altitude de 13,24 m), RS, Brasil, durante o período de fevereiro de 1993 a janeiro de 1995.

Em cada área ecológica foram instaladas duas armadilhas, eqüidistantes $30 \mathrm{~m}$ e suspensas a $1,20 \mathrm{~m}$ do solo. Em uma das armadilhas de cada local, foi colocado um recipiente plástico com $11 \mathrm{~cm}$ de diâmetro e $7 \mathrm{~cm}$ de profundidade contendo $250 \mathrm{~g}$ de fígado bovino e na outra, vísceras de galinha (exceto coração, fígado e estômago mecânico), ambos em decomposição, além de água para manter as iscas umedecidas. Estas, antes de serem usadas, permaneciam de cinco a sete dias em frascos de vidros fechados, em temperatura ambiente, para acelerar o processo inicial de decomposição. As armadilhas permaneceram instaladas durante todo o período 
experimental, removendo-se os dípteros duas vezes por semana.

As coletas foram efetuadas simultaneamente nas três áreas de captura, com substituição de $50 \%$ da isca a cada 10 dias, mantendo-se o teor de umidade. Os califorídeos foram previamente mortos com "spray" inseticida à base de piretróide, acondicionados em frascos e levados ao laboratório para identificação e quantificação. A identificação das espécies foi baseada nos trabalhos de MARILUis (1981) e DEAR (1985); os espécimes foram depositados na coleção entomológica do Museu de História Natural da Universidade Católica de Pelotas.

A abundância populacional foi estimada mediante a quantificação dos espécimes para cada espécie de Chrysomya, independente da área de captura e atrativos utilizados. Estimou-se a flutuação populacional através da média mensal de exemplares por espécie, relacionada à temperatura e à precipitação pluviométrica média mensal.

Através da análise de correlação linear simples, estudou-se a influência da temperatura e precipitação pluviométrica (média mensal) sobre a flutuação populacional das espécies. A análise estatística foi realizada pelo Sistema de Análise Estatística (SANEST) e os dados de temperatura e precipitação pluviométrica foram fornecidos pela Estação Agroclimatológica da Universidade Federal de Pelotas.

\section{RESULTADOS E DISCUSSÃO}

Foram capturados 409920 espécimes de califorídeos, sendo $85,1 \%$ pertencentes a Chrysomya. A espécie mais abundante foi C. albiceps Wiedmann, 1819 com 64,5\%, seguida por C. megacephala Fabricius, 1794 e C. putoria Wiedmann, 1830, com abundâncias respectivas de $19,7 \%$ e $0,9 \%$ (tab. I). Foram capturados ainda 60915 espécimes $(14,9 \%)$ de outros califorídeos,

Tabela I. Abundância populacional das espécies de Chrysomya capturadas em armadilhas WOT, no período de fevereiro/1993 a janeiro/1995, em Pelotas, RS, Brasil.

\begin{tabular}{lrc}
\hline & \multicolumn{2}{c}{ Abundância } \\
\cline { 2 - 3 } Espécies & Absoluta & Relativa (\%) \\
\hline Chrysomya albiceps & 264494 & 64,52 \\
Chrysomya megacephala & 80671 & 19,68 \\
Chrysomya putoria & 3840 & 0,94 \\
Outras Calliphoridae & 60915 & 14,86 \\
\hline Total & 409920 & 100,00 \\
\hline
\end{tabular}

correspondendo a Cochliomyia hominivorax (Coquerel, 1858), C. macellaria (Fabricius, 1775), Lucilia sericata (Meigen, 1826), L. eximia (Wiedmann, 1819), L. cuprina (Wiedmann, 1830), Calliphora lopesi Mello, 1962, C. vicina (Robineau-Desvoidy, 1830), Sarconesia chlorogaster (Wiedmann, 1830), Paralucilia xanthogeneiates Dear, 1985 e Hemilucilia semidiaphana (Rondani, 1850).

As três espécies de Chrysomya foram mais abundantes a temperaturas entre $14,5^{\circ} \mathrm{C}$ e $23,5^{\circ} \mathrm{C}$, cuja abundância de espécimes capturados correspondeu a $91,5 \%$ para $C$. megacephala, $95,7 \%$ para $C$. albiceps e $99,1 \%$ para $C$. putoria (tab.II). A maior abundância populacional das espécies de Chrysomya ocorreu nos meses com temperaturas entre $18,5^{\circ} \mathrm{C}$ e $23,5^{\circ} \mathrm{C}$ (outono), ou seja, nos meses de março, abril e maio, enquanto que nos meses de junho a novembro a população foi muito reduzida ou ausente. Nos meses de dezembro, janeiro e fevereiro a abundância populacional de Chrysomya representou $38 \%$ daquela verificada no outono. Os resultados são similares aos obtidos por CosTA et al. (1992), que indicaram que a população das espécies de Chrysomya, em Capão do Leão, RS, reduziu-se nos períodos com temperaturas baixas ou elevadas, alcançando pico populacional no outono. Sousa \& LINHARES (1997), ao estudarem a sazonalidade de dípteros em cadáveres de suínos na região de Campinas, SP, observaram que essas espécies foram mais abundantes nos meses mais quentes do ano.

A abundância da população de Chrysomya nos meses com temperaturas superiores a $18,5^{\circ} \mathrm{C}$ e inferiores a $23,5^{\circ} \mathrm{C}$ sugere que a temperatura é um fator importante na flutuação populacional das espécies estudadas. A análise de correlação entre a média mensal dos espécimes capturados de cada espécie e a temperatura média mensal, no mês de captura e um, dois e três meses anteriores à captura, indicaram correlação significativa $(\alpha<0,05)$. Para a população de $C$. albiceps, a temperatura influenciou os três meses anteriores à captura incluindo o mês de captura. Para $C$. megacephala e $C$. putoria, a correlação foi significativa somente para os três meses anteriores à captura (tab. III). Esta constatação está de acordo com DAJOZ (1983), que mencionou que a flutuação populacional no ambiente natural é influenciada por fatores abióticos e bióticos, sendo os fatores abióticos os mais importantes, enquanto que os bióticos exercem apenas papel secundário.

Tabela II. Abundância populacional de três espécies de Chrysomya capturadas em armadilhas WOT, relacionada com a temperatura, no período de fevereiro/1993 a janeiro/1995, em Pelotas, RS, Brasil.

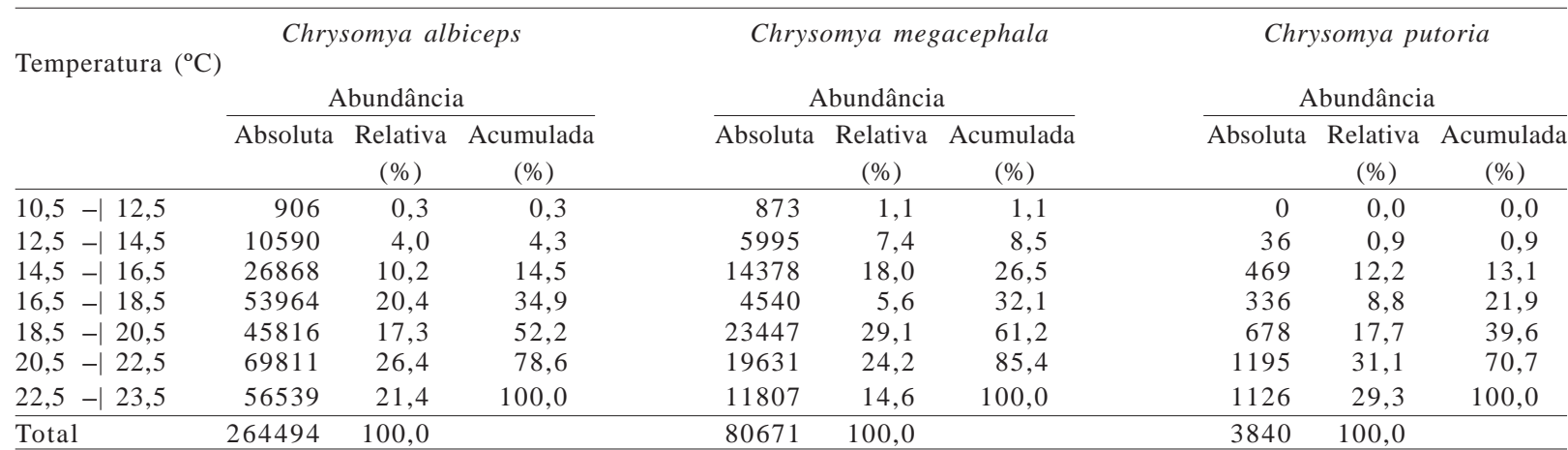



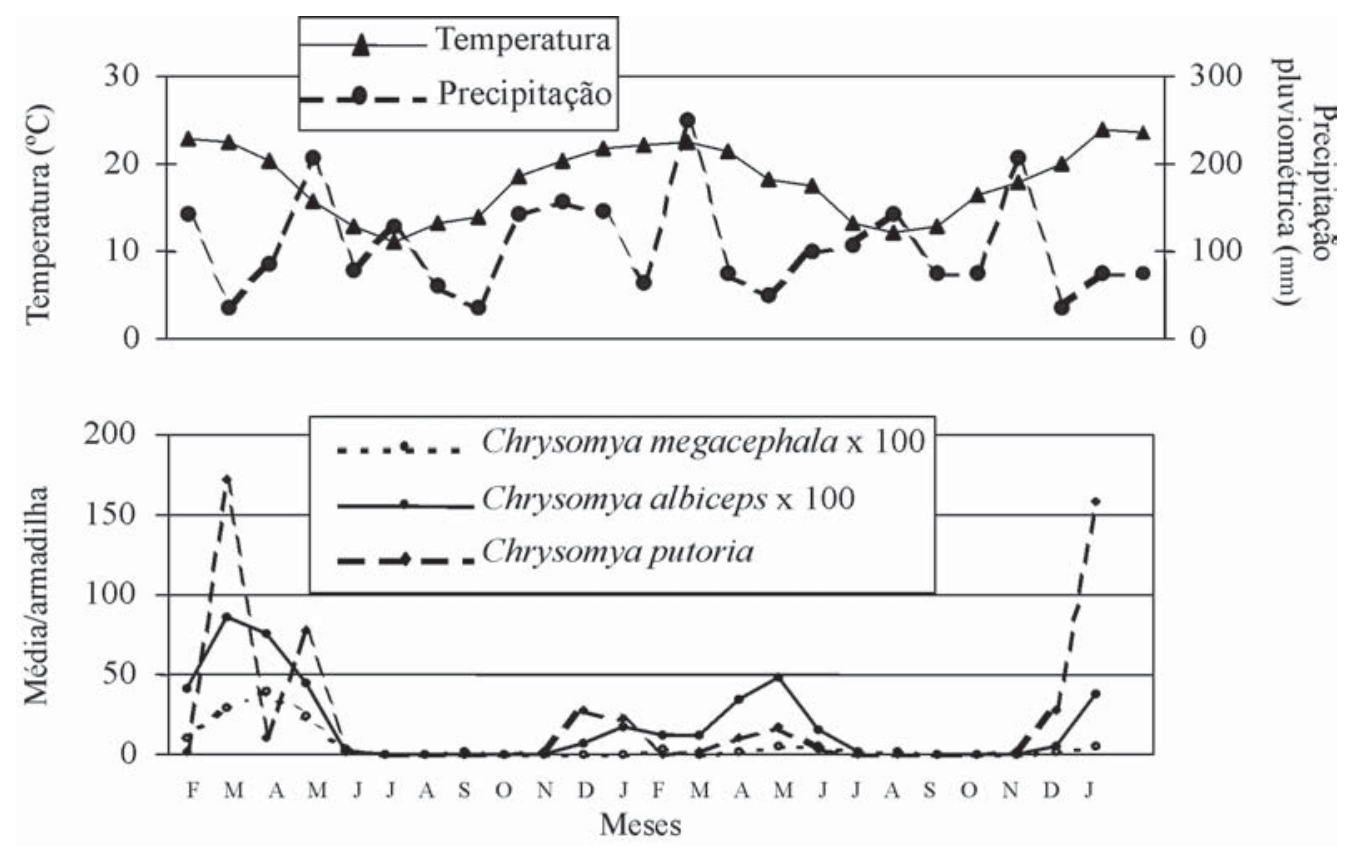

Figs. 1, 2. 1, Variação das médias mensais de precipitação pluviométrica e temperatura; 2, Flutuação populacional das espécies de Chrysomya no período de fevereiro/1993, em Pelotas, RS, Brasil.

Tabela III. Análise de correlação entre a média mensal de Chrysomya e a temperatura média mensal (TMM), no respectivo mês e com antecedência de 1,2 e 3 meses à captura, no período de fevereiro/1993 a janeiro/1995, em Pelotas, RS, Brasil (valores seguidos por asterisco $(*)$ indicam correlação significativa $(\alpha<0,05)$; variável temperatura não transformada; variável espécie transformada segundo raiz $(1+0,5) ; 0 \mathrm{M}$, TMM no respectivo mês de captura; $1 \mathrm{M}$, TMM um mês anterior à captura; $2 \mathrm{M}, \mathrm{TMM}$ dois meses anteriores à captura; $3 \mathrm{M}$, TMM três meses anteriores à captura).

\begin{tabular}{llccc}
\hline \multirow{2}{*}{ Espécies } & \multicolumn{4}{c}{ Coeficiente de Correlação } \\
\cline { 2 - 5 } & \multicolumn{4}{c}{ Meses de captura } \\
\cline { 2 - 5 } & $0,59^{*}$ & $1 \mathrm{M}$ & $2 \mathrm{M}$ & $3 \mathrm{M}$ \\
\hline Chrysomya albiceps & $0,86^{*}$ & $0,68^{*}$ \\
Chrysomya megacephala & 0,36 & $0,71^{*}$ & $0,87^{*}$ & $0,80^{*}$ \\
Chrysomya putoria & 0,46 & $0,68^{*}$ & $0,79^{*}$ & $0,61^{*}$ \\
\hline
\end{tabular}

A flutuação populacional apresentou modelo similar para as três espécies de Chrysomya (figs. 1, 2), com evidente influência da temperatura, variando apenas quanto à intensidade. $\mathrm{O}$ mesmo não ocorreu quanto à precipitação pluviométrica, que se apresentou distribuída ao longo do ano, não influenciando, segundo análise de correlação $(\alpha<0,05)$, a flutuação populacional dessas espécies. Comparando a flutuação populacional de $C$. albiceps, C. megacephala e C. putoria ao longo de dois anos, constata-se que o período de maior ocorrência e o pico populacional foram similares nos meses de outono, havendo variação apenas quanto à abundância e uma redução acentuada nos meses mais frios do ano. SousA \& LiNHARES (1997) verificaram que muitas espécies destes dípteros apresentam sazonalidade padrão e assinalam que o pico populacional das espécies de Chrysomya, no Brasil, ocorre nos meses com temperaturas superiores a $18^{\circ} \mathrm{C}$. Esse comportamento indica que as baixas temperaturas constituem fator limitante para esses dípteros, por tratar-se de espécies introduzidas, conforme Oliveira (1982), originárias da África e também devido a oferta alimentar que diminui drasticamente a temperaturas baixas.

\section{REFERÊNCIAS BIBLIOGRÁFICAS}

Baumgartner, D. L. \& Greenberg, B. 1984. The genus Chrysomya (Diptera: Calliphoridae) in the New World. Journal of Medical Entomology, Honolulu, 21(1):105113.

Broce, A. B.; Goodenough, J. L. \& Coppedge, J. R. 1977. A wind oriented trap for screworm flies. Journal of Economic Entomology, Honolulu, 70(4):413-416.

Catts, E. P. \& Goff, M. L. 1992. Forensic entomology in criminal investigations. Annual Review of Entomology, Palo Alto, 37:253-272.

Costa, P. R. P.; Wiegand, M. M. et al. 1992. Flutuação populacional das espécies de Chrysomya (Diptera: Calliphoridae) no município de Capão do Leão, RS. Arquivo Brasileiro de Medicina Veterinária e Zootecnia, Belo Horizonte, 44(4):289-296.

DAJoz, R. 1983. Ecologia geral. 4 ${ }^{\mathrm{a}}$. ed. Petrópolis, Vozes. 472p.

DEAR, J. P. 1985. A revision of the New World Chrysomya (Diptera: Calliphoridae). Revista Brasileira de Zoologia, São Paulo, 3(3):109-169.

Ferreira, M. J. M. 1978. Sinantropia de dípteros muscóideos de Curitiba, Paraná. I Calliphoridae. Revista Brasileira de Biologia, Rio de Janeiro, 38(2):445-454.

1983. Sinantropia de Calliphoridae (Diptera) em Goiânia, Goiás. Revista Brasileira de Biologia, Rio de Janeiro, 43(2): 199-210.

Greenberg, B. 1973. Flies and disease. Biology and disease transmission. Princeton, Princeton Univ. v.2, $447 \mathrm{p}$.

Guimarães, J. H.; Prado, A. P. \& Linhares, A. X. 1978. Three newly introduced blowfly species in southern Brazil (Diptera: Calliphoridae). Revista Brasileira de Entomologia, São Paulo, 22(1):53-60.

Linhares, A. X. 1981. Synantropy of Calliphoridae and Sarcophagidae (Diptera) in the city of Campinas, São Paulo, Brazil. Revista Brasileira de Entomologia, São Paulo, 25(3): 189-215.

Mariluis, J. C. 1981. Chave para la identificación de los Calliphoridae de la Republica Argentina (Diptera). Revista de la Sociedad Entomológica Argentina, Buenos Aires, 40(1-4):27-30.

Moura, M. O.; Carvalho, C. J. B. de \& Monteiro Filho, E. L. A. 1997. A preliminary analysis of insects of Medico-Legal importance in Curitiba, State of Paraná. Memórias do 
Instituto Oswaldo Cruz, Rio de Janeiro, 92:269-274.

Nuorteva, P. 1963. Synantropy of blowflies (Diptera: Calliphoridae) in Finland. Annales Entomologici Fennici, Helsinki, 29:1-49.

Oliveira, C. M B. DE. 1982. Ocorrência e flutuação populacional de três espécies do gênero Chrysomya. Pesquisa Agropecuária Brasileira, Brasilia, 30(4):497498.

Patton, E. M. 1922. Some notes of Indian Calliphoridae. Part III. Chrysomya megacephala F. (Dux esch) the common Indian blue bottles, whose larvae occasionally cause myiasis in animals and Chrysomya nigriceps sp. nov. the common blue bottle of the Nilgiris, Indian. Indian Journal of Medical Research, New Delhi, 9(3):555-560.

Schoof, H. F.; Mall, C. A. \& Savage, E. P. 1954. Fly production sources in urban communities. Journal of Economic Entomology, New York, 47:245-253.

Silveira-Neto, S.; NaKano, O. et al. 1976. Manual de ecologia dos insetos. São Paulo, Ceres. 419p.

Sousa, A. M. \& Linhares, A. X. 1997. Diptera and Coleoptera of potential forensic importance in southeastern Brazil: relative abundance e seasonality. Medical and Veterinary Entomology, Oxford, 11:8-12.

Stewart, M. A. \& Roesler, E. B. 1942. The seasonal distribution of miiases producing Diptera. Journal of Economic Entomology, New York, 35:408-411.

Vianna, E. E. S.; Brum, J. G. W. et al. 1997. Sinanthropy of Calliphoridae (Diptera) in Pelotas, Rio Grande do Sul State, Brazil. Revista Brasileira de Parasitologia Veterinária, São Paulo, 7(2):141-147.

Von Zuben, C. J.; Reis, S. F. et al. 1993. Dynamics of a mathematical model of Chrysomya megacephala (Diptera: Calliphoridae). Journal of Medical Entomology, Lanham, 30(2):443-448.

Zumpt, F. 1965. Myiasis in man and animals in the Old World. London, Butterworhs. 267p. 